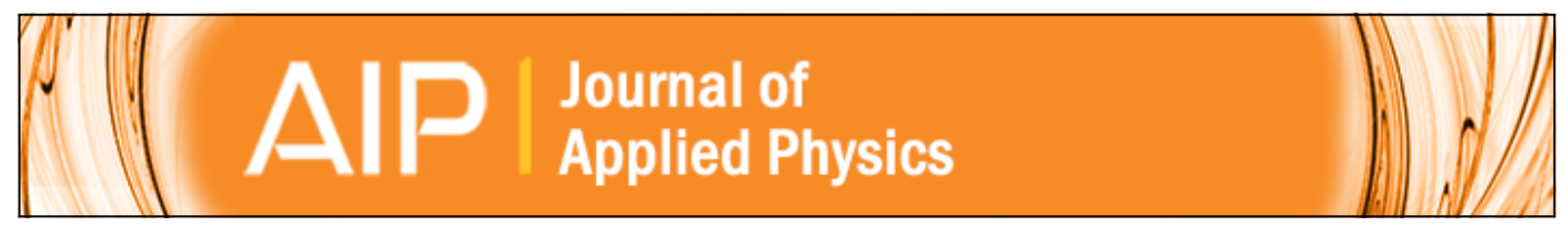

\title{
Magnetoelectric effect in crystallographically textured BaTiO 3 films deposited on ferromagnetic metallic glass foils
}

Zhiguang Wang, Li Yan, Yaodong Yang, Jie-Fang Li, Jaydip Das, Anton L. Geiler, Aria Yang, Yajie Chen, V. G. Harris, and D. Viehland

Citation: Journal of Applied Physics 109, 034102 (2011); doi: 10.1063/1.3544352

View online: http://dx.doi.org/10.1063/1.3544352

View Table of Contents: http://scitation.aip.org/content/aip/journal/jap/109/3?ver=pdfcov

Published by the AIP Publishing

\section{Articles you may be interested in}

Ferroelectric and ferromagnetic properties in $\mathrm{BaTiO} 3$ thin films on $\mathrm{Si}(100)$

J. Appl. Phys. 116, 094103 (2014); 10.1063/1.4894508

Enhanced magnetoelectric effect in La0.67Sr0.33MnO3/PbZr0.52Ti0.48O3 multiferroic nanocomposite films with a SrRuO3 buffer layer

J. Appl. Phys. 113, 164106 (2013); 10.1063/1.4803057

Enhancement in magnetoelectric response in Co Fe $204-\mathrm{Ba} \mathrm{Ti} O 3$ heterostructure

Appl. Phys. Lett. 92, 062911 (2008); 10.1063/1.2841048

Magnetoelectric $\mathrm{Co} F e ~ 20$ 4/Pb ( Zr $0.52 \mathrm{Ti} 0.48$ ) O 3 double-layer thin film prepared by pulsed-laser deposition

Appl. Phys. Lett. 88, 013111 (2006); 10.1063/1.2162262

Magnetoelectric CoFe $2 \mathrm{O} 4-\mathrm{Pb}$ ( $\mathrm{Zr}, \mathrm{Ti}$ ) O 3 composite thin films derived by a sol-gel process

Appl. Phys. Lett. 86, 122501 (2005); 10.1063/1.1889237

\section{MIT LINCOLN} LABORATORY CAREERS

Discover the satisfaction of innovation and service to the nation
- Space Control

- Air \& Missile Defense

- Communications Systems \& Cyber Security

- Intelligence, Surveillance and

Reconnaissance Systems

$$
\begin{aligned}
& \text { - Advanced } \\
& \text { Electronics } \\
& \text { - Tactical Systems } \\
& \text { " Homeland } \\
& \text { Protection } \\
& \text { - Air Traffic Control }
\end{aligned}
$$

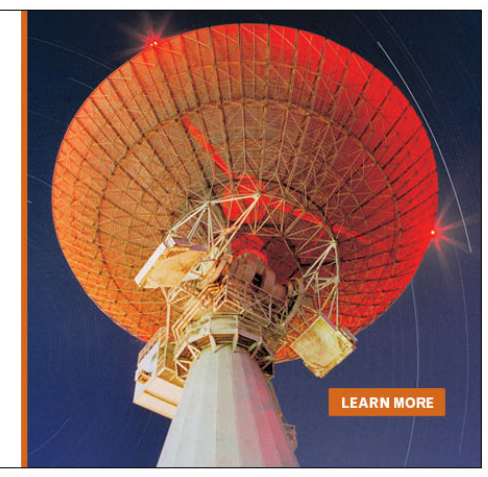




\title{
Magnetoelectric effect in crystallographically textured $\mathrm{BaTiO}_{3}$ films deposited on ferromagnetic metallic glass foils
}

\author{
Zhiguang Wang, ${ }^{1, a)}$ Li Yan, ${ }^{1}$ Yaodong Yang, ${ }^{1}$ Jie-Fang Li, ${ }^{1}$ Jaydip Das, ${ }^{1}$ Anton L. Geiler, ${ }^{2}$ \\ Aria Yang, ${ }^{2}$ Yajie Chen, ${ }^{2}$ V. G. Harris,${ }^{2}$ and D. Viehland ${ }^{1}$ \\ ${ }_{1}^{1}$ Department of Materials Science and Engineering, Virginia Tech, 306 Holden Hall, Blacksburg, \\ Virginia 24061, USA \\ ${ }^{2}$ Department of Electrical and Computer Engineering, Center for Microwave Magnetic Materials and \\ Integrated Circuits, Northeastern University, Boston, Massachusetts 02115, USA
}

(Received 22 November 2010; accepted 14 December 2010; published online 2 February 2011)

\begin{abstract}
We demonstrate a significant control of the polarization response under an applied magnetic field for a magnetoelectric (ME) heterostructure. This structure was comprised of a $2 \mu \mathrm{m}$ thick ferroelectric $\mathrm{BaTiO}_{3}$ (BTO) film deposited on flexible ferromagnetic metallic glass foil (25 $\mu \mathrm{m}$ thick). Au was used as a buffer layer to control BTO growth orientation, and to protect the metallic glass from oxidation. x-ray diffraction and scanning electron microscopy demonstrated the successful growth of well-crystallized BTO films with a high degree of (111) orientation on the amorphous metallic glass foils. Well-defined polarization (P-E) and magnetization (M-H) hysteresis loops confirmed the coexistence of ferroelectric and ferromagnetic properties. A ME voltage coefficient of about $\sim 60 \mathrm{mV} / \mathrm{cm}$ Oe was measured. (C) 2011 American Institute of Physics. [doi:10.1063/1.3544352]
\end{abstract}

\section{INTRODUCTION}

The magnetoelectric (ME) effect in both single phase and composite materials has been a topic of much research in the past decade, both from scientific and application perspectives. ${ }^{1-6}$ Studies have revealed the simultaneous presence of polarized electronic and magnetic systems, where there is a coupling between systems. Accordingly, an electronic polarization $(\mathrm{P})$ can be induced under the application of a magnetic field $(\mathrm{H})$, and vice versa, a magnetization polarization $(\mathrm{M})$ can be realized under the application of an electric field (E). Unfortunately, in single phase ME materials the electronic configurations that favor magnetization are antagonistic to those that favor electronic polarization. ${ }^{7}$ $\mathrm{Cr}_{2} \mathrm{O}_{3}, \mathrm{BiFeO}_{3}$, and rear earth oxides are the most popular single phase ME materials but all of them suffer from very low Curie temperature and at least one phase would be antiferroic at room temperature thus the ME coupling effect is very weak. After decades of research, single phase materials have yet to be found that have simultaneous high $\mathrm{P}$ and $\mathrm{M}$, and thus high ME coupling effect at room temperature. Engineered composite ME materials have become the only means, to date, by which to achieve a sizable and useful ME effect.

Composite ME ceramic materials with two phase powders mixed together are first studied but the low resistivity of ferromagnetic phase make it very difficult to fully polarize the ferroelectric phase and ME effect is not very high. Castel et al. ${ }^{8,9}$ have successfully made $\mathrm{BaTiO}_{3}$ (BTO)/Ni nanocomposites and measured dependence of permittivity of BTO phase with applied $\mathrm{H}$ due to the ME coupling effect happened in the interface of magnetostrictive $\mathrm{Ni}$ and piezoelectric BTO. Laminate composites of magnetostrictive and piezoelectric layers have been shown to have much higher ME

${ }^{a)}$ Electronic mail: zgwang@vt.edu. coefficients than those of single-phase materials or particulate composites. Magnetostrictive layer will change its shape and dimension under applied magnetic field, and the strain would then be transferred to piezoelectric layer through elastic interaction. And if you measure the dielectric polarization change in the piezoelectric layer you can get a changing output voltage response with the applied magnetic field, that is ME coupling effect. The highest ME coefficient to date has been reported in Metglas $/ \mathrm{Pb}(\mathrm{Zr}, \mathrm{Ti}) \mathrm{O}_{3}$ laminate structures: ${ }^{10,11}$ which have ME voltage coefficients as high as $\alpha_{\mathrm{ME}}=20 \mathrm{~V} / \mathrm{cm}$ Oe. Based on these ME structures, Dong et $a l .{ }^{10}$ have developed highly sensitive, passive magnetic-field sensors that operate at low frequencies with noise floors on the order of $10^{-11} \mathrm{~T} / \sqrt{\mathrm{Hz}}$ at $\mathrm{f}=1 \mathrm{~Hz}$ Ref. 12 when using a simple operational amplifier detection method. However, the disadvantage of bulk laminated composites is obvious: First, the size is large, making integration with $\mathrm{Si}$ or other substrates difficult; second, the best magnetostrictive materials are metallic alloys, making the use of ceramic cofiring and packaging improbable; third, the dissimilar nature of these layers requires epoxy for bonding component layers, providing a less than ideal interface to facilitate the transmittal of strain between magnetostrictive and piezoelectric layers. Furthermore, epoxy has problems such as aging, especially when working under resonant conditions. The presence of epoxy can also decrease the ME coefficient due to interfacial relaxation. ${ }^{13}$

Recently, Zhao et al. ${ }^{13}$ created PZT/Galfenol heterostructures on Si cantilevers. After etching away the Si substrate, they demonstrated that mechanical clamping effects from the substrate on the PZT/Galfenol heterostructure were greatly mitigated, notably improving $\alpha_{\mathrm{ME}}$ relative to that of the unetched Si structures. Brintlinger et al. ${ }^{14}$ mechanically released BTO/Galfenol layers from $\mathrm{SrTiO}_{3}$ (STO) substrates by a focused ion beam method, and observed magnetic domain wall movement in the $\mathrm{FeGa}$ layer due to the strain 

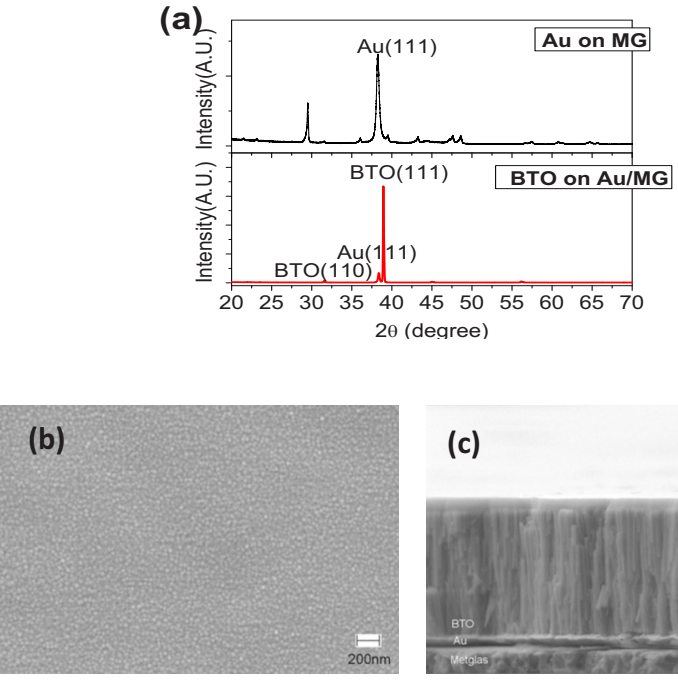

(c)

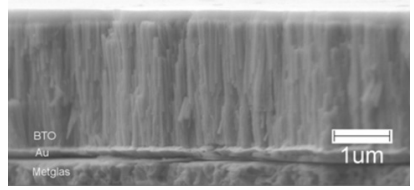

FIG. 1. (Color online) Crystallization and microstructural results for BTO on Au/Metglas (a) XRD line scan; (b) SEM image taken from a top view; and $(\mathrm{c})$ cross section view.

transferred from the BTO layer under the application of an electric field. The ME effect for these heteroepitaxial ME composites is a magnetoelastoelectric effect, just as is the case for laminated ME composites. For these examples, structures must be grown on a substrate suitable to be mechanically released. However, microelectromechanical systems technologies, typically used for this purpose, suffer from low fabrication rates and are often incompatible with $\mathrm{Si}$ processing technology.

Here, we report the direct deposition of BTO films on ferromagnetic metallic glass (MG) foils by the pulsed laser deposition (PLD) technique. Commercial, $25 \mu \mathrm{m}$ thick, $\mathrm{MG}$ foils were obtained from Vacuumschmelze (Germany). These MG foils had piezomagnetic coefficients of $\mathrm{d}_{33, \mathrm{~m}}$ $=4 \mathrm{ppm} / \mathrm{Oe}$, which were three to four times higher than that for Terfenol-D and Galfenol. ${ }^{15,16}$

\section{EXPERIMENT DETAILS}

PLD was used to deposit BTO layers on the MG foils. First, Au was sputtered as a buffer layer on the MG in order to achieve highly ordered BTO layers. Sputtering was performed for a duration of $10 \mathrm{~min}$. Once the Au formed a continuous layer on the amorphous MG substrates, a secondary grain growth, driven by the anisotropic surface free energy, ${ }^{17}$ resulted in a (111) texture. PLD of BTO was accomplished using a $\mathrm{KrF}$ excimer laser, $\lambda=248 \mathrm{~nm}$. To obtain a high atomic mobility, ${ }^{18}$ as required for crystallization of oriented BTO, a laser energy density of $20 \mathrm{~J} / \mathrm{cm}^{2}$ was uti- lized. This allowed for the ejection of high energy particles from the BTO target, thus enabling the use of a lower deposition temperature and minimizing the oxidation of the $\mathrm{MG}$ foil substrates. The size of the laser spot was $\sim 2 \mathrm{~mm}^{2}$, and the distance between substrate and target was $8 \mathrm{~cm}$. Deposition was carried out in a 90 mTorr oxygen atmosphere with a base vacuum of $10^{-6}$ Torr. During the first $10 \mathrm{~min}$ of BTO layer deposition, a laser frequency of $10 \mathrm{~Hz}$ was used and a temperature of $300{ }^{\circ} \mathrm{C}$ to prevent melting of the Au layer and subsequent $\mathrm{MG}$ oxidation. Next, the substrate temperature was increased to $600{ }^{\circ} \mathrm{C}$ and the deposition cycle was continued for a duration of $\sim 90 \mathrm{~min}$. The surface and cross sectional morphologies of the BTO films were then studied using a LEO (Zeiss) 1550 Schottky field-emission scanning electron microscopy (SEM). The crystal structures of BTO and the MG substrate were characterized using a Philips $X$ 'pert high resolution $x$-ray diffractometer. The magnetic properties of the MG layers were measured with a Lakeshore 7300 Series vibrating sample magnetometer (VSM) System at room temperature.

\section{RESULTS AND DISCUSSION}

\section{A. Crystallization and growth orientation}

$\mathrm{X}$-ray diffraction (XRD) results before and after ablation of the BTO target are compared in Fig. 1(a). Before ablation, the (111) peak of the textured Au layer dominates the spectrum with smaller diffraction features identified to arise from the partially crystallized MG substrate. After ablation, a peak appears that is much more intense than other diffraction features: The d-spacing $(\mathrm{d} \approx 0.23 \mathrm{~nm})$ of this peak is consistent with the (111) diffraction peak $(\mathrm{d}=0.231 \mathrm{~nm})$ of the BTO structure. This supports the growth of highly oriented BTO films on MG substrates. One may assume a local cube-oncube epitaxial relationship between the Au buffer layer and the BTO film, such that: (111)BTO\|(111)Au| (amorphous)MG. Good in-plane alignment and crystal quality of the BTO film is confirmed by the measurement of a full width at half maximum (FWHM), value of $0.14^{\circ}$ for the (111) BTO diffraction peak. Other low intensity peaks, along different zone axes, correspond to other reflections from BTO and are also indicated in Fig. 1(a).

Figure 1(b) shows an SEM image of a top view of our $\mathrm{BTO} / \mathrm{Au} / \mathrm{MG}$ heterostructure. One sees BTO formed as a uniform layer with a grain size of about $40 \mathrm{~nm}$. From the cross sectional image presented as Fig. 1(c), one sees that $\mathrm{Au}$ formed as a dense and uniform interface between MG and BTO with a thickness of about $60 \mathrm{~nm}$. This thickness was sufficient to prevent oxidation of the MG upon subsequent
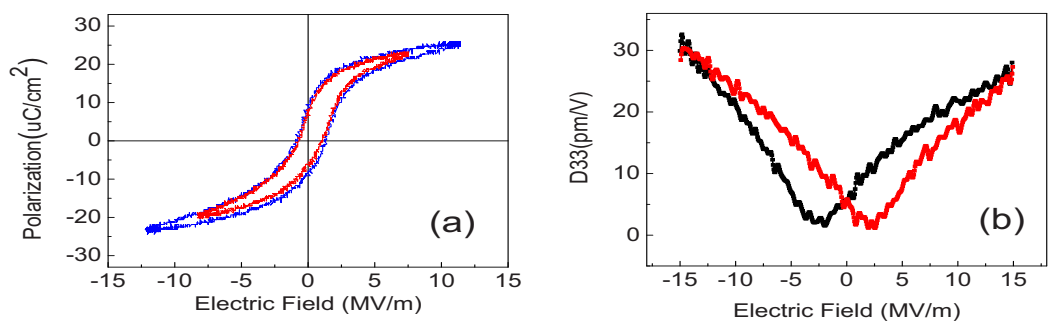

FIG. 2. (Color online) Ferroelectric properties of BTO on Au/Metglas: (a) polarization hysteresis loop; (b) piezoelectric hysteresis loop. 

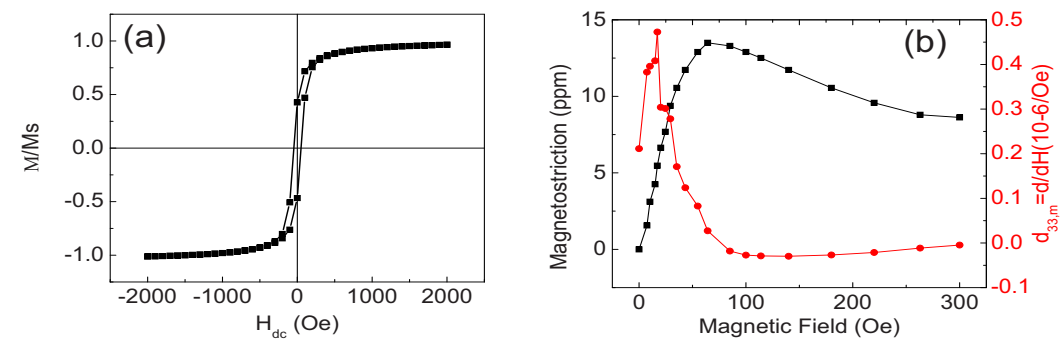

FIG. 3. (Color online) (a) Magnetic properties of Metglas with BTO grown on top: (a) normalized magnetization vs applied dc magnetic bias field; and (b) magnetostrictive and piezomagnetic properties as a function of dc magnetic bias.

deposition of BTO while maintaining (111) orientation of the BTO. The BTO/Au/MG structure was golden in color; however, BTO/MG appeared as black indicating the possible oxidation of MG. The BTO layer thickness reaches $\sim 2 \mu \mathrm{m}$ after $2 \mathrm{~h}$ of deposition, with a high-density columnar microstructure of grain size $40 \mathrm{~nm}$ visible in cross section.

\section{B. Ferroelectric and ferromagnetic properties}

The ferroelectric properties BTO/Au/MG samples were characterized by a polarization hysteresis measurement using a triangular signal of frequency $100 \mathrm{kHz}$. Figure 2 shows a well-defined ferroelectric hysteresis loop, with a saturation polarization, $\mathrm{P}_{\mathrm{s}} \approx 25 \mu \mathrm{C} / \mathrm{cm}^{2}$, and a remnant polarization, $\mathrm{P}_{\mathrm{r}} \approx 7.5 \mu \mathrm{C} / \mathrm{cm}^{2}$. Both of these values are notably larger than those of BTO deposited on Pt buffered Si. ${ }^{19,20}$ The electric coercive field was $12.5 \mathrm{kV} / \mathrm{cm}$, which was correspondingly much smaller than that of BTO thin films on STO or $\mathrm{Si}^{21}$ After the polarization measurements, the BTO films were polarized in the out-of-plane direction and the piezoelectric properties were measured. Figure 2(b) shows $d_{33, p}$ as a function of electric field. A well-defined butterfly-like loop can be seen with a saturation value for the out-of-plane piezoelectric coefficient value of $\mathrm{d}_{33, \mathrm{p}} \approx 30 \mathrm{pm} / \mathrm{V}$ : which again was much larger than that of the oriented BTO films on $\mathrm{Au} / \mathrm{Si}^{22}$ One of the contributing factors for the enhanced $\mathrm{d}_{33, \mathrm{p}}$ value is that the BTO layers of the present study are constrained only by a thin $(25 \mu \mathrm{m})$ foil, where as BTO films on $\mathrm{Si}$ substrates are constrained by thick $(500 \mu \mathrm{m}) \mathrm{Si}$ substrate and thus the constrain effect is much larger.

The ferromagnetic properties of the MG layer were measured at room temperature using a VSM. Figure 3(a) shows a typical M-H loop for the BTO/Au/MG after the high temperature deposition process. The saturation field was $\sim 400$ Oe, and the coercive field was $\sim 60$ Oe. Figure 3(b) shows the magnetostriction $(\lambda)$ of MG measured by a strain gauge method. At about 80 Oe dc magnetic field, the magnetostriction reached a value of about $14 \mathrm{ppm}$. The maximum value of $\mathrm{d}_{33, \mathrm{~m}}$ was $\sim 0.5 \mathrm{ppm} / \mathrm{Oe}$ at a dc magnetic field of 200 Oe. This value is comparable with that of Terfenol-D, which has the highest magneostriction but is still only $1 / 8$ of that of MG in the as-received condition. These results strongly suggest that the MG amorphous foil has undergone partial, if not total, crystallization during the high temperature film processing.

\section{ME coupling coefficient measurement}

Finally, the ME voltage coefficient was measured, using the structure and measurement method as shown in Fig. 4(a). The Au buffer layer served as the bottom electrode for the BTO film. A patterned Au top electrode was then sputtered onto the surface of the BTO film. Electrical contacts from the sample were made by gold wire bonding. Details of the measurement method were reported previously, ${ }^{23}$ which involved the use of a lock-in amplifier. A magnet was used as the dc magnetic signal source. A $20 \mathrm{~Hz}$ ac magnetic signal was then applied along the long axis of the heterostructure. This resulted in an induced strain, via magnetostriction, along the MG foil. The induced strain from the MG was transmitted to the BTO layer, resulting in a voltage that was detected by a lock-in amplifier. Figure 4(b) shows the ME voltage coefficient as a function of dc $\mathrm{H}$ bias. A maximum value of $\alpha_{\mathrm{ME}}$ $\approx 55 \mathrm{mV} / \mathrm{cm}$ Oe was found corresponding to a dc bias field of $20 \mathrm{Oe}$. This value is notably larger than heteroepitaxial and self-assembled ME composites on various substrates. ${ }^{23}$ The reason for this higher value compared with that of oxide ferrites is because of the higher value $d_{33, m}$ of the magnetostrictive alloy foil. Another reason is that the substrate itself is magnetostrictive, thus the structure does not have a large constraint stress, as is unavoidable in ME heteroepitaxial layers or (2-2) structure thin films grown on nonmagnetostrictive substrates.

\section{CONCLUSIONS}

In summary, we have successfully epitaxial grown (111) oriented BTO thin films on magnetostrictive MG foils. A buffer of Au was first sputtered on the MG to protect it from oxidation. Substantial ferroelectric and ferromagnetic properties were obtained from the BTO layers and MG foils. An ME voltage coefficient of $\alpha_{\mathrm{ME}} \approx 55 \mathrm{mV} / \mathrm{cm} \mathrm{Oe}$ was achieved due to strain coupling between the piezoelectric BTO layer and the magnetostrictive MG foil. Our direct

(a)
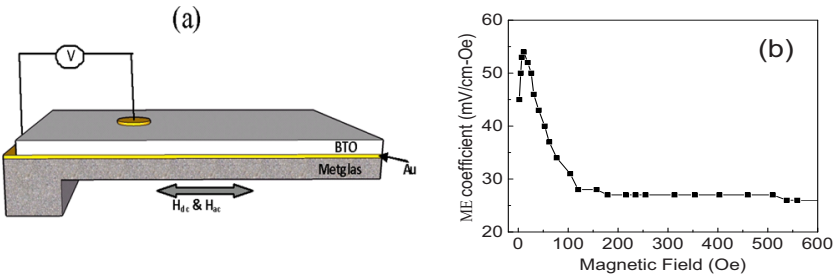

FIG. 4. (Color online) (a) Schematic of the BTO/Au/Metglas multilayer structure and measurement setup; and (b) ME voltage coefficient as a function of dc magnetic field. 
deposition of highly oriented BTO layers on MG foils with comparable thicknesses eliminates substrate clamping of the ME heterostructure, enhancing the piezoelectric and piezomagnetic coefficients of the respective layers toward values closer to those of their bulk forms, and thus enabling improved magnetoelectricity.

\section{ACKNOWLEDGMENTS}

The authors would like to thanks the Air Force Office of Scientific Research (Grant No. FA 9550-06-1-0410) and Department of Energy (Contract No. DE.-AC02-98CH10886) for financial support of this work.

${ }^{1}$ J. Wang, J. Neaton, H. Zheng, V. Nagarajan, S. Ogale, B. Liu, D. Viehland, V. Vaithyanathan, D. Schlom, U. Waghamare, N. A. Spadlin, K. M. Rabe, M. Wuttig, and R. Ramesh, Science 299, 1719 (2003).

${ }^{2}$ H. Zheng, J. Wang, S. E. Lofland, Z. Ma, L. Mohaddes-Ardabili, T. Zhao, L. Salamanca-Riba, S. R. Shinde, S. B. Ogale, F. Bai, D. Viehland, Y. Jia, D. G. Gchlom, M. Wuttig, A. Roytburd, and R. Ramesh, Science 303, 661 (2004).

${ }^{3}$ S. Ryu, J. H. Park, and H. M. Jang, Appl. Phys. Lett. 91, 142910 (2007).

${ }^{4}$ M. Ziese, A. Bollero, I. Panagiotopoulos, and N. Moutis, Appl. Phys. Lett. 88, 212502 (2006).

${ }^{5}$ H. C. He, J. Wang, J.-P. Zhou, and C.-W. Nan, Adv. Funct. Mater. 17, 1333 (2007).

${ }^{6}$ J. Zhai, Z. Xing, S. Dong, J. Li, and D. Viehland, J. Am. Ceram. Soc. 91, 351 (2008).

${ }^{7}$ C. Ederer and N. A. Spaldin, Curr. Opin. Solid State Mater. Sci. 9, 128
(2005).

${ }^{8}$ V. Castel and C. Brosseau, Appl. Phys. Lett. 92, 233110 (2008).

${ }^{9}$ V. Castel, C. Brosseau, and J. Ben Youssef, J. Appl. Phys. 106, 064312 (2009).

${ }^{10}$ D. Shuxiang, Z. Junyi, X. Zengping, L. Jie-Fang, and D. Viehland, Appl. Phys. Lett. 86, 102901 (2005).

${ }^{11}$ Z. Xing, J. Zhai, J. Gao, J. Li, and D. Viehland, IEEE Electron Device Lett. 30, 445 (2009).

${ }^{12}$ J. Das, J. Gao, Z. Xing, J. F. Li, and D. Viehland, Appl. Phys. Lett. 95, 092501 (2009).

${ }^{13}$ P. Zhao, Z. Zhao, D. Hunter, R. Suchoski, C. Gao, S. Mathews, M. Wuttig, and I. Takeuchi, Appl. Phys. Lett. 94, 243507 (2009).

${ }^{14}$ T. Brintlinger, S.-H. Lim, K. H. Baloch, P. Alexander, Y. Qi, J. Barry, J. Melngailis, L. Salamanca-Riba, I. Takeuchi, and J. Cumings, Nano Lett. 10, 1219 (2010).

${ }^{15}$ M. B. Moffett, A. E. Clark, M. Wun-Fogle, J. Linberg, J. P. Teter, and E. A. McLaughin, J. Acoust. Soc. Am. 89, 1448 (1991).

${ }^{16}$ A. E. Clark, M. Wun-Fogle, J. B. Restorff, T. A. Lograsso, and J. R. Cullen, IEEE Trans. Magn. 37, 2678 (2001).

${ }^{17}$ C. C. Wong, H. I. Smith, and C. V. Thompson, Appl. Phys. Lett. 48, 335 (1986).

${ }^{18}$ J. Schwarzkopf and R. Fornari, Prog. Cryst. Growth Charact. Mater. 52, 159 (2006).

${ }^{19}$ Y. W. Cho, S. K. Choi, and G. Venkata Rao, Appl. Phys. Lett. 86, 202905 (2005).

${ }^{20}$ B. Dkhil, E. Defaÿ, and J. Guillan, Appl. Phys. Lett. 90, 022908 (2007).

${ }^{21}$ W. Pompe, X. Gong, Z. Suo, and J. S. Speck, J. Appl. Phys. 74, 6012 (1993).

${ }^{22}$ Y. Yang, J. Yao, J. Li, J. Das, and D. Viehland, Thin Solid Films 518, 5806 (2010).

${ }^{23}$ L. Yan, Z. Xing, Z. Wang, T. Wang, G. Lei, J. Li, and D. Viehland, Appl. Phys. Lett. 94, 192902 (2009) 